\title{
Changes in Etiologies of Hospitalized Patients with Liver Cirrhosis in Beijing 302 Hospital from 2002 to 2013
}

\author{
Binxia Chang, ${ }^{1}$ Baosen Li, ${ }^{1}$ Ying Sun, ${ }^{1}$ Guangju Teng, ${ }^{1}$ Ang Huang, ${ }^{1}$ Jin Li, ${ }^{2}$ \\ and Zhengsheng Zou ${ }^{1}$ \\ ${ }^{1}$ Center for Diagnosis and Treatment of Non-Infectious Liver Disease, Beijing 302 Hospital, Beijing 100039, China \\ ${ }^{2}$ Department of Medical Administration, Beijing 302 Hospital, Beijing 100039, China \\ Correspondence should be addressed to Jin Li; lijin302@hotmail.com and Zhengsheng Zou; zszou302@163.com
}

Received 26 July 2017; Accepted 25 October 2017; Published 19 November 2017

Academic Editor: Ju Qiu

Copyright (c) 2017 Binxia Chang et al. This is an open access article distributed under the Creative Commons Attribution License, which permits unrestricted use, distribution, and reproduction in any medium, provided the original work is properly cited.

\begin{abstract}
Background. Over the last 20 years, the prevalence of hepatitis B virus (HBV) infection in China has decreased gradually due to the application of a national HBV vaccination program. In contrast, the prevalence of alcoholic liver disease (ALD), nonalcoholic fatty liver disease, autoimmune liver disease, and drug-induced liver injury has markedly increased. Methods. We conducted a retrospective review of 82,562 hospitalized patients diagnosed with liver cirrhosis in Beijing 302 Hospital from 2002 to 2013. Results. The top four etiologies of cirrhosis were HBV, HCV, ALD, and autoimmune liver disease. The percentage of HBV cirrhosis decreased from $81.53 \%$ in 2002 to $66.0 \%$ in 2013, whereas the frequency of alcoholic cirrhosis increased from $3.34 \%$ in 2002 to $8.40 \%$ in 2013. Females $(84.34 \%$ ) accounted for the majority of cirrhotic patients with autoimmune liver diseases. Males accounted for $80.16 \%$ of HBV cirrhosis patients and $98.02 \%$ of alcoholic cirrhosis patients. Conclusion. In Beijing 302 Hospital, the top four etiologies of cirrhosis were HBV, HCV, ALD, and autoimmune liver disease. Over the last 12 years, the prevalence of HBV cirrhosis has decreased gradually, whereas that of alcoholic cirrhosis has increased significantly.
\end{abstract}

\section{Introduction}

Recent years, viral hepatitis, especially chronic hepatitis B $(\mathrm{CHB})$, is still a main reason of liver-related morbidity and mortality in China. While the prevalence of hepatitis B virus (HBV) infections fell from 10\% to 7\% between 1992 and 2006 as a result of a national HBV vaccination program [1]. However, due to increased national production and consumption of alcoholic beverages in China, alcoholic liver disease (ALD) is emerging as a leading cause of chronic liver disease. According to published studies, the point prevalence of ALD ranges from $2.3 \%$ to $6.1 \%$, with a median prevalence of $4.5 \%$ in the Chinese population [2, 3]. In addition to ALD, the prevalence of autoimmune liver disease and druginduced liver injury is increasing in China.

The aim of the present study was to investigate the variation in the disease spectrum of hospitalized patients diagnosed with liver cirrhosis in Beijing 302 Hospital between 2002 and 2013.

\section{Study Population and Methods}

2.1. Patients. This was a retrospective analysis of patients admitted to Beijing 302 Hospital, which is a large tertiary hospital specialized in liver diseases in Beijing. From 2002 to $2013,82,562$ patients diagnosed with liver cirrhosis were admitted to Beijing 302 Hospital. The diagnosis of cirrhosis and associated etiologies was based on clinical practice guidelines [4-9]. Patient data were obtained from the hospital's medical records. After active treatment, amelioration of symptoms, physical findings, and complications, in addition to improvements in abnormal liver function or coagulation function, was considered an improvement. 
TABLE 1: The etiologies of 82,562 hospitalized cirrhotic patients from 2002 to 2013 .

\begin{tabular}{lccc}
\hline Etiology of cirrhosis & Cases & $\begin{array}{c}\text { Constituent } \\
\text { ratio (\%) }\end{array}$ & Rank \\
\hline Hepatitis B & 58,742 & 71.15 & 1 \\
Hepatitis C & 9627 & 11.66 & 2 \\
Alcoholic liver disease & 5517 & 6.68 & 3 \\
Autoimmune liver disease & 4080 & 4.94 & 4 \\
Cryptogenic cirrhosis & 2681 & 3.25 & 5 \\
Hepatitis B overlapping C & 1119 & 1.36 & 6 \\
Drug-induced liver injury & 548 & 0.66 & 7 \\
Hepatolenticular degeneration & 128 & 0.16 & 8 \\
Vascular obstruction disease & 33 & 0.04 & 9 \\
Nonalcoholic fatty liver & 32 & 0.04 & 10 \\
disease & 28 & 0.03 & 11 \\
Bilharziasis & 24 & 0.03 & 12 \\
Cardiac cirrhosis & 3 & 0.00 & 13 \\
Malnutritional cirrhosis & & &
\end{tabular}

2.2. Statistical Analysis. Continuous variables with a normal distribution were expressed as the mean \pm standard deviation $($ mean $\pm S D)$. Data that were not normally distributed were expressed as the median (interquartile range). An analysis of variance and SNK test were used to compare nonparametric and parametric continuous variables. Categorical variables were expressed as frequencies, with percentages. The categorical variables were analyzed by an $\mathrm{R} \times \mathrm{C}$ chi-square test or the Kruskal-Wallis test. Data were analyzed using SPSS version 18.0 for Windows (SPSS, Chicago, IL). Tests were two-sided, and a probability $(P)$ value of less than 0.05 was considered statistically significant.

2.3. Ethical Approval. The study was approved by the ethics committee of Beijing 302 Hospital, and the study conformed to the Helsinki Declaration of 1977. Written informed consent was obtained from all the patients and volunteers.

\section{Results}

3.1. Etiologies. The etiologies of the 82,562 hospitalized cirrhotic patients are shown in Table 1. The top four etiologies of cirrhosis were $\mathrm{HBV}, \mathrm{HCV}, \mathrm{ALD}$, and autoimmune liver diseases. Among 4080 patients with autoimmune liver cirrhosis, there were 2225 cases of autoimmune hepatitis $(\mathrm{AIH})$ and 1855 cases of primary biliary cirrhosis (PBC).

3.2. Changes in Etiologies of Cirrhosis in the Last 12 Years. The most common etiologies of cirrhosis were HBV, $\mathrm{HCV}, \mathrm{ALD}$, and autoimmune liver disease, with a total of 77,966 patients diagnosed with these diseases in the past 12 years. Cirrhosis caused by them accounted for $94.43 \%$ of cases, as discussed in this paper. Supplemental Table 1 in Supplementary Material available online at https:/doi.org/ $10.1155 / 2017 / 5605981$ and Figure 1 show changes in the disease spectrum of cirrhosis patients in the past 12 years. The

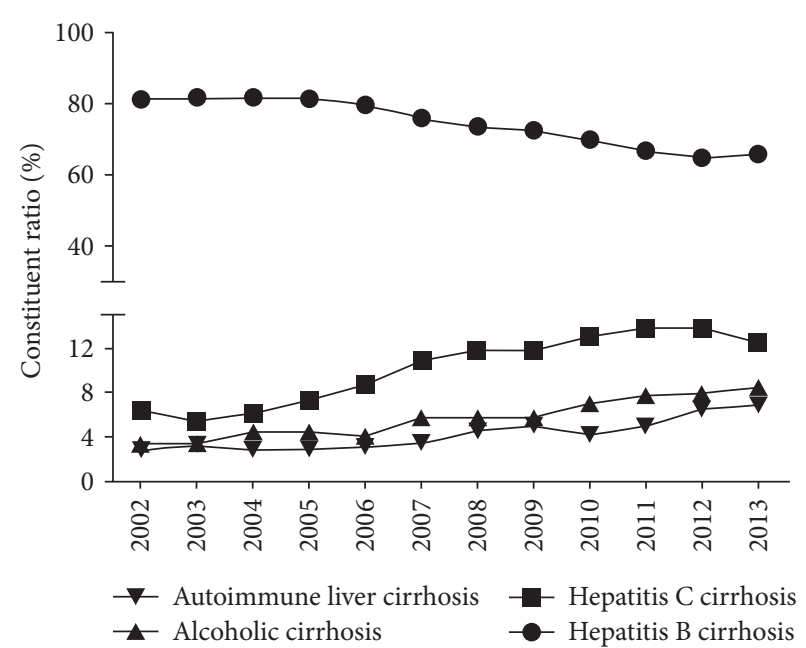

FIgURE 1: Changes in etiologies of cirrhosis from 2002 to 2013. For the hospitalized patients in Beijing 302 Hospital, the percentage of HBV cirrhosis decreased from $81.53 \%$ in 2002 to $66.00 \%$ in 2013 . Cirrhosis due to HCV, ALD, and autoimmune liver disease increased gradually over time. Alcoholic cirrhosis increased 2.5 times from $3.34 \%$ in 2002 to $8.40 \%$ in 2013 .

percentage of $\mathrm{HBV}$ cirrhosis decreased from $81.53 \%$ in 2002 to $66.00 \%$ in 2013. Cirrhosis due to HCV, ALD, and autoimmune liver disease increased gradually over time. Alcoholic cirrhosis increased 2.5 times from 3.34\% in 2002 to $8.40 \%$ in 2013 .

3.3. Gender Distribution of the Cirrhosis Patients. Figure 2 shows the gender distribution of the cirrhosis patients. Females accounted for the majority of patients with autoimmune liver cirrhosis. The percentage of female and male patients with HCV was similar. However, males accounted for the majority of $\mathrm{HBV}$ and alcoholic cirrhosis patients. The gender distribution of the cirrhosis groups was significantly different $(P<0.01)$.

3.4. Age of the Cirrhosis Groups. The ages of the different cirrhosis groups are indicated in Table 2. Most patients with alcoholic cirrhosis and HBV were younger than 50 years, whereas most patients with HCV cirrhosis and autoimmune liver cirrhosis were older than 50 years. There was a marked difference among groups with the $P$ value less than 0.01 .

3.5. Geographic Origin of the Patients in the Different Cirrhosis Groups. The geographic origins were divided into North China, East China, Central China, South China, Northeast China, Northwest China, and Southwest China. Figure 3 and Supplemental Table 2 show the distribution and origins of the cirrhosis groups. Most cirrhotic patients came from North China. Most patients with HBV cirrhosis, HCV cirrhosis, alcoholic cirrhosis, and autoimmune liver cirrhosis were from North China and Northeast China. However, due to the selection bias in our hospital, this distribution cannot be considered to be representative of all of China. 


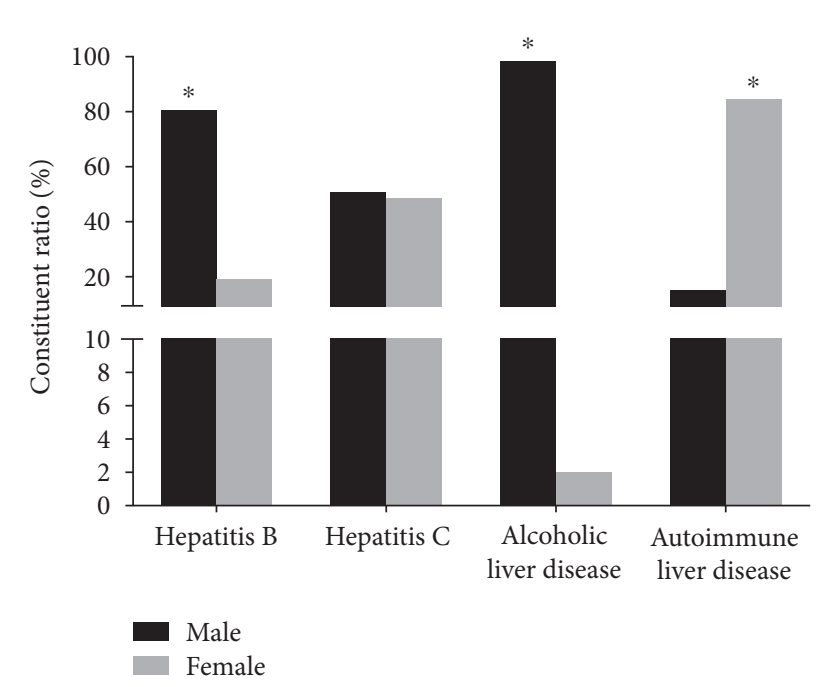

Figure 2: Gender distribution of the cirrhosis patients. For the inpatients in Beijing 302 Hospital, females accounted for the majority of patients with autoimmune liver cirrhosis. The percentage of female and male patients with HCV was comparative. Males accounted for the majority of $\mathrm{HBV}$ and alcoholic cirrhosis patients $\left({ }^{*} P<0.01\right)$.

TABle 2: Age of the cirrhosis groups.

\begin{tabular}{lccc}
\hline & Cases & Mean & $P$ value \\
\hline Hepatitis B cirrhosis & 58,742 & $48.19 \pm 11.14$ & \\
Hepatitis C cirrhosis & 9627 & $56.73 \pm 10.12$ & \multirow{2}{*}{0.000} \\
Alcoholic cirrhosis & 5517 & $49.62 \pm 10.23$ & \\
Autoimmune liver cirrhosis & 4080 & $56.57 \pm 12.21$ & \\
\hline
\end{tabular}

Compared with hepatitis B cirrhosis or alcoholic cirrhosis, $P<0.01$.

3.6. Prognosis of the Patients in the Different Cirrhosis Groups. Table 3 shows the prognosis of the patients in the different cirrhosis groups. After active treatment, more than $70 \%$ of $\mathrm{HBV}$ and HCV cirrhosis patients and nearly $80 \%$ of alcoholic and autoimmune liver cirrhosis patients showed improvements. When compared with the other cirrhosis groups, there were significant differences $(P<0.01)$.

\section{Discussion}

Cirrhosis refers to end-stage liver disease, which is caused by multiple factors. It is associated with various complications, including ascites, upper gastrointestinal hemorrhage, hepatic encephalopathy, and spontaneous peritonitis. It is a complex disease, with a poor prognosis. Primary hepatic cancer may occur in some cirrhosis patients. Others may require a liver transplantation because of liver cancer or decompensation of liver function.

The disease spectrum of cirrhosis in China differs from that in other countries, where cirrhosis is mainly caused by ALD and hepatitis C. For example, Moreau et al. [10] reported that alcoholic cirrhosis accounted for $66.6 \%$ of all cirrhosis cases in France, whereas hepatitis C cirrhosis, alcoholic cirrhosis overlapping with viral hepatitis, and hepatitis

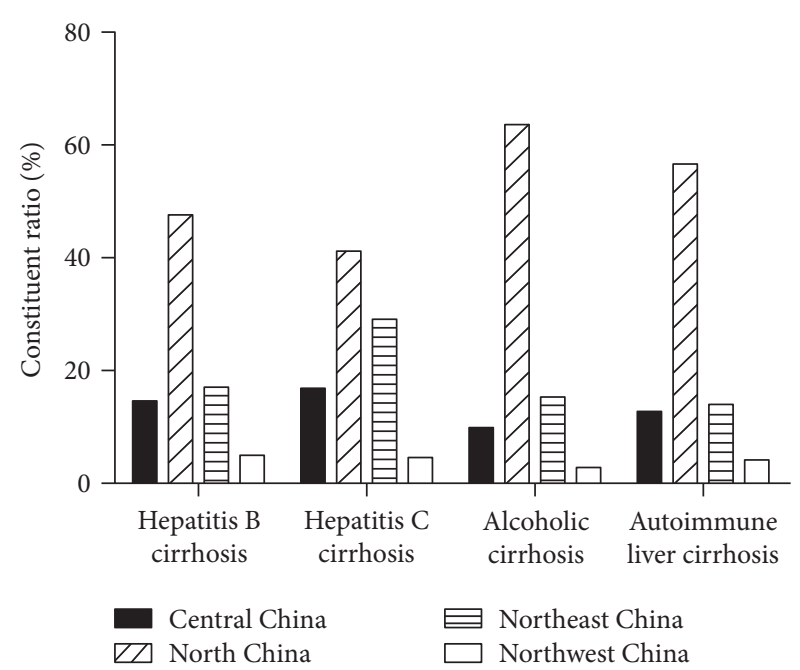

FIGURE 3: Geographic origin of the patients in the different cirrhosis groups. In this research, most patients with HBV cirrhosis, HCV cirrhosis, alcoholic cirrhosis, and autoimmune liver cirrhosis were from North China and Northeast China. North China includes Beijing, Tianjin, Hebei, Neimenggu, and Shanxi province. East China includes Shandong, Shanghai, Jiangsu, Anhui, Zhejiang, and Fujian province. Henan, Hubei, Hunan, and Jiangxi province are included in Central China. Guangdong, Guangxi, and Hainan are classified as South China. Northeast China consists of Heilongjiang, Jilin, and Liaoning. Northwest China comprises Ningxia, Qinghai, Shanxi, Xinjiang, and Gansu province. Southwest China includes Sichuan, Xizang, Yunnan, Guizhou, and Chongqing.

B cirrhosis accounted for $16 \%, 14.7 \%$, and $2.7 \%$ of cases, respectively. Haukeland et al. [11] reported that among 1264 patients diagnosed with cirrhosis from January 1999 to March 2004, 53\% of cases were caused by ALD and the remaining cases were due to hepatitis (12\%), autoimmune liver disease $(12 \%)$, hemochromatosis (4\%), and nonalcoholic fatty liver disease (3\%). The etiology in $16 \%$ of cases was unknown. However, based on a high incidence of diabetes mellitus, the authors concluded that cirrhosis in these cases may have been caused by nonalcoholic steatohepatitis. ALD was reported to be responsible for more than $50 \%$ of cirrhosis cases in European countries. In contrast, in our research, ALD was responsible for less than $10 \%$ of cases of cirrhosis. However, as alcohol consumption continues to increase in China, the incidence of alcoholic cirrhosis will also likely increase.

There is a high incidence of HBV infection in China. According to an epidemiological investigation of hepatitis $\mathrm{B}$ in 2006, the carrying rate of HBsAg was $7.18 \%$ in the population from 1 year to 59 years [12]. There are around 93 million individuals with chronic HBV infection, and about 20 million of these are chronic hepatitis patients [13]. Cirrhosis is primarily the result of $\mathrm{HBV}$ infection. In the present study, $71.15 \%$ of the 82,562 cirrhosis cases were caused by hepatitis B. The HBV infection rate has fallen dramatically in Chinese children since the introduction of the $\mathrm{HBV}$ vaccine inoculation program, and the carrying rate of HBsAg today is only $0.96 \%$ among children under 5 years [12]. The incidence of hepatitis $\mathrm{B}$ cirrhosis also continues to decrease annually as 
Table 3: Prognosis of the patients in the different cirrhosis groups.

\begin{tabular}{|c|c|c|c|}
\hline & Improvement & Inefficacy or death & $P$ value \\
\hline Hepatitis B cirrhosis & $41,736(71.05 \%)$ & $17,006(28.95 \%)$ & \multirow{4}{*}{0.000} \\
\hline Hepatitis C cirrhosis & $6997(72.68 \%)$ & $2630(27.32 \%)$ & \\
\hline Alcoholic cirrhosis & $4365(79.12 \%)$ & $1152(20.88 \%)$ & \\
\hline Autoimmune liver cirrhosis & $3261(79.93 \%)$ & $819(20.07 \%)$ & \\
\hline
\end{tabular}

a result of the availability of effective antiviral drugs, such as nucleoside analogs and interferon. As shown in the present study, the percentage of hepatitis B cirrhosis declined from $81.53 \%$ in 2002 to $66.00 \%$ in 2013.

Since HBV and HCV were found, several decades have passed. This explains why most of the patients with hepatitis $\mathrm{B}$ and $\mathrm{C}$ cirrhosis in the present study were middle aged to old. The incidence of HCV can be expected to decrease further as a result of strict screening of blood products and using effective antiviral drugs.

With improvements in socioeconomic conditions, alcohol consumption has increased. According to one report in China, the production of alcohol rose from 7.113 million tons in 1984 to 30.699 million tons in 2001 [14]. There have been no nationwide epidemiological investigations of ALD. However, a regional epidemiological study found that the drinking population and incidence of ALD showed an upward trend. A survey of North China reported that the ratio of intemperants increased from $0.21 \%$ in the 1980 s to $14.3 \%$ in the 1990s [15]. Since the beginning of this century, in some Chinese province, the drinking population has increased from $26.98 \%$ to $43.4 \%$ and the incidence of ALD in adults has increased from $4.3 \%$ to $6.5 \%[2,16,17]$. A multiplecenter study indicated that from 2000 to 2004, the hospitalized ratio of ALD to all liver diseases was $2.7 \%, 2.9 \%, 3.0 \%$, $3.6 \%$, and $4.4 \%$, respectively [18]. From 2002 to 2013, there were 7422 hospitalized ALD patients in Beijing 302 Hospital, with the ratio to other liver diseases rising from $1.74 \%$ in 2002 to $4.60 \%$ in 2013 [19]. This result was similar to that of a multiple-center study in China, which will be discussed in another paper [19]. In this study, the percentage of hospitalized alcoholic cirrhosis patients increased from $3.34 \%$ in 2002 to $8.40 \%$ in 2013. Alcoholic cirrhosis had become the third most common cause of cirrhosis after hepatitis B and C cirrhosis.

The results of this paper were in accord with the real situation in China (i.e., the drinking population mainly consisted of middle-aged males). In addition, the majority of patients with autoimmune liver cirrhosis were middle-aged females. In the past, due to the absence of an effective diagnostic method, autoimmune hepatitis was difficult to diagnose. However, in recent years, it has been paid more and more attention. In the present study, the majority of cirrhosis cases were patients from North China and Northeast China. However, due to selection bias in the hospital, this distribution is not representative of China.

In this study, the top four etiologies of cirrhosis in Beijing 302 Hospital were HBV, HCV, ALD, and autoimmune liver disease. Although the prevalence of hepatitis B cirrhosis has decreased, the prevalence of alcoholic cirrhosis has increased gradually. A nationwide multiple-center study is needed to detect changes in etiologies of hospitalized patients with liver cirrhosis in the whole country.

\section{Conflicts of Interest}

The authors declare that they have no conflicts of interest.

\section{Authors' Contributions}

Binxia Chang and Baosen Li contributed equally to this paper.

\section{Acknowledgments}

This paper was supported by the National Natural Science Foundation of China (NSFC) with Grant nos. 81370530 and 81600467.

\section{References}

[1] H. L. Chan and J. Jia, "Chronic hepatitis B in Asia-new insights from the past decade," Journal of Gastroenterology and Hepatology, vol. 26, Supplement 1, pp. 131-137, 2011.

[2] S. L. Chen, X. D. Meng, B. Y. Wang, and G. Q. Xiang, “An epidemiologic survey of ALD in some cities of Liaoning province," Shiyong Ganzangbing Zazhi, vol. 13, pp. 428-430, 2010.

[3] J. H. Yao, Q. D. Zhao, P. F. Xiong et al., "Investigation of ALD in ethnic groups of Yuanjiang county in Yunman," Weichangbingxue He Ganbingxue Zazhi, vol. 20, pp. 11371139, 2011.

[4] Chinese Society of Hepatoloty, Fatty Liver and Alcoholic Liver Disease Study Group, "The diagnosis and treatment guideline of alcoholic liver disease," Linchuang Gandanbing Zazhi, vol. 26, pp. 229-232, 2010.

[5] R. S. O'Shea, S. Dasarathy, A. J. McCullough, Practice Guideline Committee of the American Association for the Study of Liver Diseases, and Practice Parameters Committee of the American College of Gastroenterology, "Alcoholic liver disease,” Hepatology, vol. 51, no. 1, pp. 307-328, 2010.

[6] European Association for the Study of the Liver, "EASL clinical practical guidelines: management of alcoholic liver disease," Journal of Hepatology, vol. 57, no. 2, pp. 399-420, 2012.

[7] Chinese Society of Hepatoloty and Chinese Society of Infecrious Diseases, Chinese Medical Association, "The prevention and treatment guidelines of chronic hepatitis B (2010)," Shiyong Ganzangbing Zazhi, vol. 14, pp. 81-89, 2011.

[8] Chinese Society of Hepatoloty, Chinese Society of Infecrious and Parasitic Diseases, Chinese Medical Association, "The prevention and treatment guidelines of hepatitis C," Chuanranbing Xinxi, vol. 17, pp. 9-14, 2004. 
[9] European Association for the Study of the Liver, "EASL clinical practice guidelines: management of cholestatic liver diseases," Journal of Hepatology, vol. 51, pp. 237-267, 2009.

[10] R. Moreau, P. Delègue, F. Pessione et al., "Clinical characteristics and outcome of patients with cirrhosis and refractory ascites," Liver International, vol. 24, pp. 457-464, 2004.

[11] J. W. Haukeland, I. Lorgen, L. T. Schreiner et al., "Incidence rates and causes of cirrhosis in a Norwegian population," Scandinavian Journal of Gastroenterology, vol. 42, no. 12, pp. 1501-1508, 2007.

[12] X. Liang, S. Bi, W. Yang et al., "Evaluation of the impact of hepatitis B vaccination among children born during 1992-2005 in China," The Journal of Infectious Diseases, vol. 200, no. 1, pp. 39-47, 2009.

[13] L. FM and H. Zhuang, "Management of hepatitis B in China," Chinese Medical Journal, vol. 122, pp. 3-4, 2009.

[14] Z. Hui, "Epidemiology of alcoholic liver disease," Chinese Journal of Hepatology, vol. 11, p. 412, 2003.

[15] Y. M. Li, W. X. Chen, C. H. Yu et al., "The epidemiological investigation of alcoholic liver disease in Zhejiang province," Chinese Journal of Hepatology, vol. 11, pp. 647-649, 2003.

[16] X. L. Lu, M. Tao, J. Y. Luo, Y. Geng, H. L. Zhao, and P. Zhao, "The epidemiological investigation of drinking and liver disease," Chinese Journal of Hepatology, vol. 10, pp. 467-468, 2002.

[17] S. L. Huang, S. Q. Dai, X. H. Zhang, Y. J. Yu, M. L. Tan, and C. G. Yi, "The epidemiological investigation of alcoholic liver disease in Hunan province," Zhongguo Yishi Zazhi, vol. 7, pp. 426-427, 2005.

[18] National investigation group of alcoholic liver disease, "National multiple investigation and analysis of alcoholic liver disease," Chinese Journal of Digestion, vol. 27, pp. 31-34, 2007.

[19] B. X. Chang, B. S. Li, A. Huang et al., "Changes of four common non-infectious liver diseases for the hospitalized patients in Beijing 302 hospital from 2002 to 2013," Alcohol, vol. 54, pp. 61-65, 2016. 


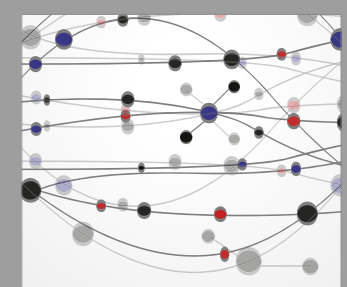

The Scientific World Journal
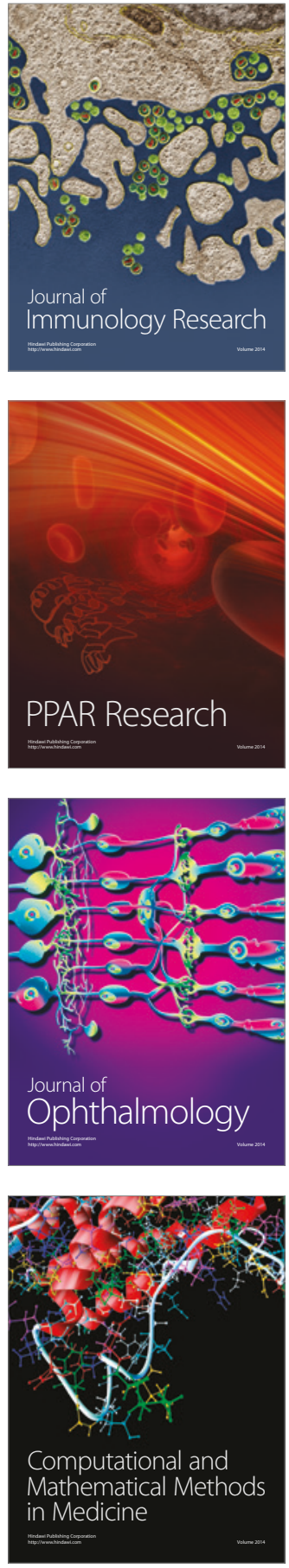

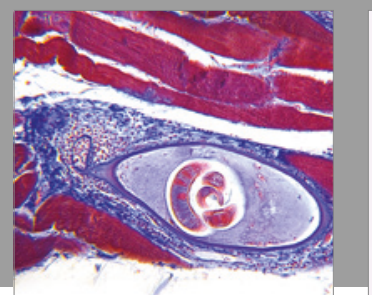

Gastroenterology Research and Practice
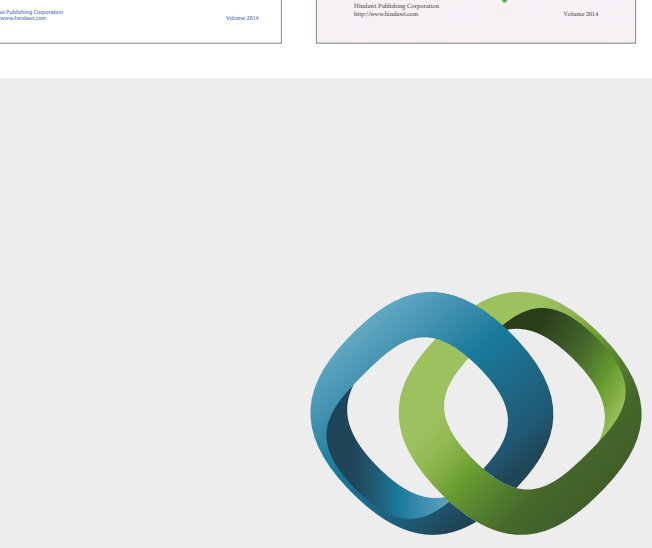

\section{Hindawi}

Submit your manuscripts at

https://www.hindawi.com
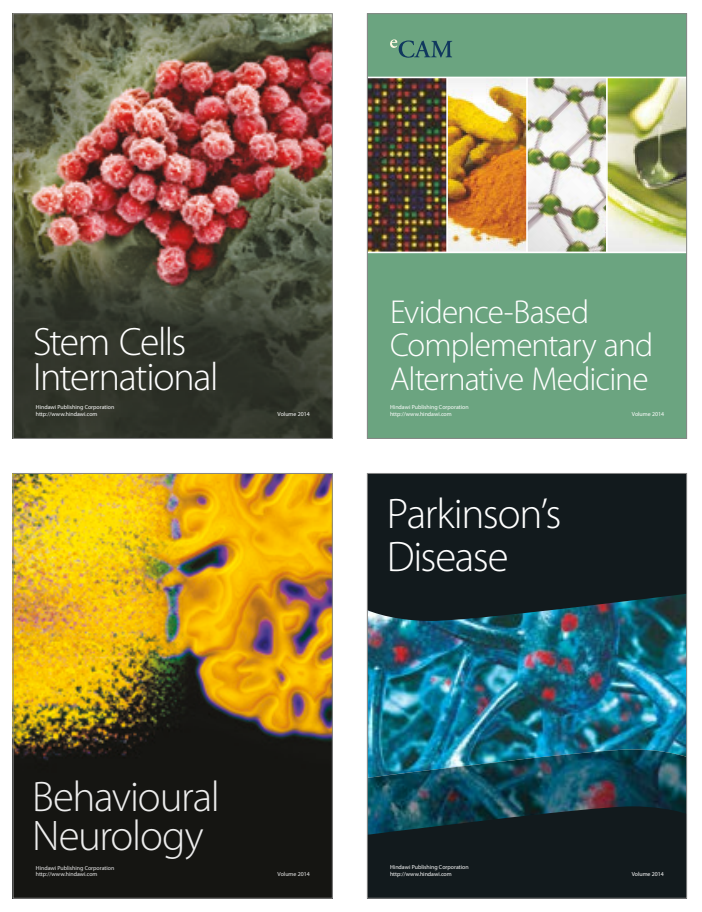
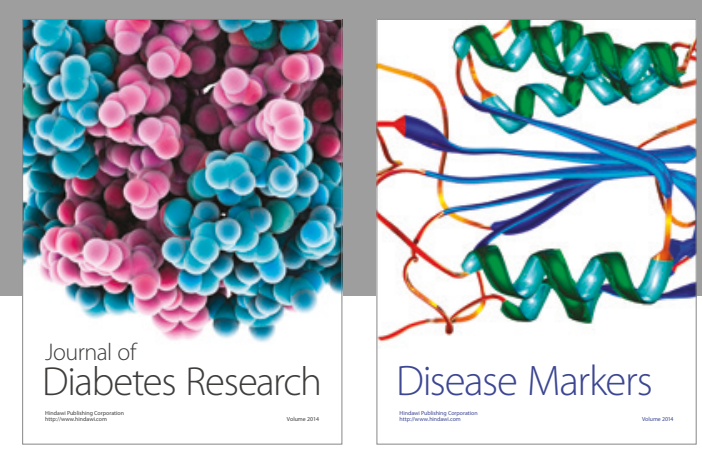

Disease Markers
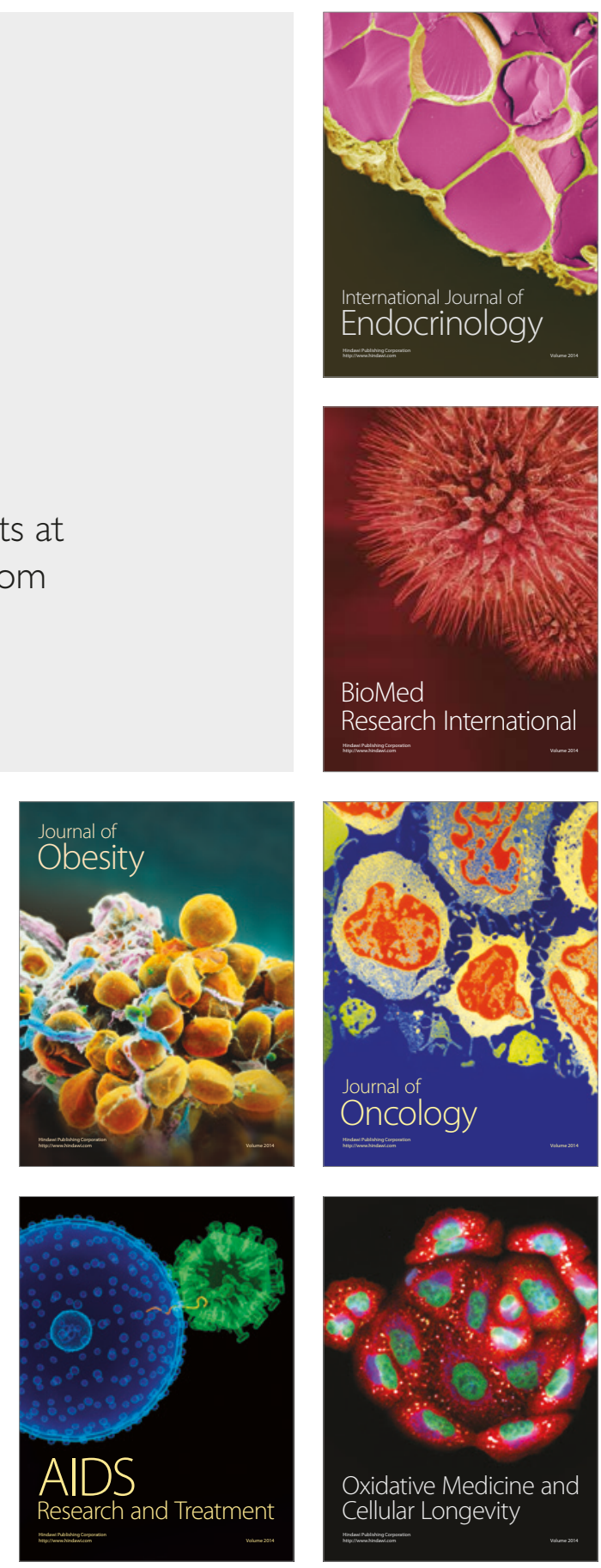